\title{
Power of PTEN/AKT: Molecular switch between tumor suppressors and oncogenes (Review)
}

\author{
YINGQIU XIE $^{1 *}$, SANZHAR NAIZABEKOV $^{1 *}$, ZHANLIN CHEN $^{2}$ and TURSONJAN TOKAY ${ }^{3}$ \\ ${ }^{1}$ Department of Biology, Nazarbayev University, School of Science and Technology, Astana 010000, Republic of Kazakhstan; \\ ${ }^{2}$ Lipscomb University, Lipscomb Academy, Nashville, TN 37204, USA; ${ }^{3}$ Center for Life Sciences, \\ National Laboratory Astana, Nazarbayev University, Astana 010000, Republic of Kazakhstan
}

Received July 21, 2015; Accepted April 29, 2016

DOI: $10.3892 / \mathrm{ol} .2016 .4636$

\begin{abstract}
An increasing amount of evidence has shown that tumor suppressors can become oncogenes, or vice versa, but the mechanism behind this is unclear. Recent findings have suggested that phosphatase and tensin homolog (PTEN) is one of the powerful switches for the conversion between tumor suppressors and oncogenes. PTEN regulates a number of cellular processes, including cell death and proliferation, through the phosphoinositide 3-kinase/protein kinase B/mammalian target of rapamycin (PI3K/AKT/mTOR) pathway. Furthermore, a number of studies have suggested that PTEN deletions may alter various functions of certain tumor suppressor and oncogenic proteins. The aim of the present review was to analyze specific cases driven by PTEN loss/AKT activation, including aberrant signaling pathways and novel drug targets for clinical application in personalized medicine. The findings illustrate how PTEN loss and/or AKT activation switches MDM2-dependent p53 downregulation, and induces conversion between oncogene and tumor suppressor in enhancer of zeste homolog 2, BTB domain-containing 7A, alternative reading frame $2, \mathrm{p} 27$ and breast cancer 1 , early onset, through multiple mechanisms. This review highlights the genetic basis of complex drug targets and provides insights into the rationale of precision cancer therapy.
\end{abstract}

\section{Contents}

1. Introduction

2. PTEN loss switches MDM2-dependent p53 downregulation through ARF

Correspondence to: Dr Yingqiu Xie, Department of Biology, Nazarbayev University, School of Science and Technology, 53 Kabanbay Batyr Avenue, Astana 010000, Republic of Kazakhstan E-mail: xieautumnus@gmail.com

${ }^{*}$ Contributed equally

Key words: PTEN, tumor suppressor, oncogene, molecular switch
3. PTEN loss/AKT activation switches EZH2 from a tumor suppressor to an oncogene

4. Pten loss switches ZbTb7a from an oncogene to a tumor suppressor

5. PTEN loss switches ARF function through sumoylation

6. PTEN loss/AKT activation switches $p 27$ from a tumor suppressor to an oncogene

7. PTEN loss/AKT activation switches $B R C A l$ from a tumor suppressor to an oncogenic resistant gene in radiation therapy

8. Conclusion and prospective outlook

\section{Introduction}

Phosphatase and tensin homolog (PTEN), a lipid phosphatase, is one of the frequently mutated or deleted tumor suppressor genes during cancer progression (1). In particular, it is deleted in $>50 \%$ of prostate cancer cases. PTEN is a well-known tumor suppressor protein that regulates a number of cellular processes, including cell death and proliferation, through the phosphoinositide 3-kinase/protein kinase B/mammalian target of rapamycin (PI3K/AKT/mTOR) pathway $(2,3)$. However, PTEN function is phosphatase-independent, as well as being dependent upon events in the nucleus $(4,5)$. It has been found that inactivated PTEN can switch oncogenes to oncosuppressors in certain mouse models, which suggests a dependence on the genetic context. In other words, PTEN inactivation may change the progression of disease from an aggressive state to safe and vice versa. In the present review, examples of altered function in the p53, enhancer of zeste homolog 2 (EZH2), alternative reading frame 2 (ARF), zinc finger and $B T B$ domain-containing 7A (ZBTB7A), p27 and breast cancer 1, early onset (BRCA1) proteins by PTEN loss/AKT activation signaling will be discussed.

\section{PTEN loss switches MDM2-dependent p53 downregula- tion through ARF}

The first and classical example of PTEN inactivation as a switch is the modulation of tumor suppressor p53 (6). p53 and PTEN control cell death and proliferation, and they are often expressed simultaneously in various types of tumor $(7,8)$. Due 
to its short half-life, the suppressor function of p53 heavily relies on this stabilization (9). In normal cells, p53 levels are kept at insignificant levels by ubiquitin-mediated proteolysis. PTEN and p53 may form a positive feedback loop. In detail, p53 can upregulate PTEN by binding to the PTEN promoter, thereby activating PTEN transcription (8). Mechanistically, PTEN inhibits AKT-mediated MDM2 phosphorylation to prevent MDM2 from translocation into the nucleus to degrade p53 (10). Thus, wild-type PTEN can stabilize p53. It can be stated that AKT activation with PTEN deletion may result in the rapid degradation of p53, leading to further PTEN-dependent tumorigenesis (11).

However, in mouse embryonic fibroblasts (MEFs) and a mouse model of prostate cancer, it has been found that PTEN loss does not induce p53 degradation or instability (12). On the contrary, Pten loss can also upregulate p53, which leads to Pten loss-induced cellular senescence through p53, and to a certain degree, restricted tumor growth (13). Mechanistically, PTEN loss induces ARF elevation and elevated ARF may degrade MDM2, thereby stabilizing p53 (14,15). Indeed, in MEFs, acute loss of Pten did not decrease p53 stability, but actually increased stability, with co-upregulation of p19 ${ }^{\text {Arf }}$ (ARF in mouse) (13). Thus, PTEN loss can also induce elevation of p53 as a molecular switch by regulating p53 stability through the ARF-MDM2 pathway. However, the mechanism via which PTEN can induce ARF upregulation remains unclear. Moreover, the method by which ARF and AKT compete for the regulation of MDM2 for the stability of p53 also requires further investigation.

\section{PTEN loss/AKT activation switches $E Z H 2$ from a tumor suppressor to an oncogene}

Epigenetic regulators are a relatively new class of therapeutic target for cancer treatment. The enhancer of zeste homolog (EZH2), a catalyst of polycomb repressive complex 2 (PRC2), is a well-known epigenetic regulator that plays oncosuppressive roles by silencing gene expression via its histone methyltransferase activity (16). Moreover, it silences transcription by trimethylating $\mathrm{H} 3$ histone on lysine 27 (16). Nevertheless, EZH2 expression correlates to the progression of prostate cancer, particularly to that of castration-resistant prostate cancer (CRPC) (17).

A recent study has reported that $\mathrm{EZH} 2$ can act as an oncogenic protein. The oncogenic function of EZH2 is independent of its role as a transcriptional repressor in cases of CRPC (17). This functional change requires phosphorylation of EZH2 by AKT, as well as an intact methyltransferase domain. Study results have shown that EZH2 phosphorylation at serine 21 can easily change its function from a PRC2 catalyst to an androgen receptor transcriptional co-activator, as it is mediated by the PI3K/AKT pathway directly and indirectly (17). These findings indicate that the oncogenic activity of EZH2 in CRPC is not dependent on its polycomb-related oncosuppressive function. Furthermore, the findings indicate that it is possible to develop inhibitors that may specifically target the activation of EZH2 without altering its PRC2 repressive function. Recent studies have suggested that PTEN loss correlates with EZH2 elevation in the invasion and metastasis of gallbladder adenocarcinoma (18). Moreover,
PTEN loss downregulates p16 expression to decrease cellular senescence, thereby increasing cell proliferation, an oncogenic process. This process is induced through the EZH2 elevation-mediated methylation change of the p16 promoter. In other words, PTEN loss may upregulate EZH2 for the inhibition of cell cycle arrest (19). Thus, PTEN loss/AKT activation may switch EZH2 function.

\section{Pten loss switches $Z b T b 7 a$ from an oncogene to a tumor suppressor}

ZBTB7A (also known as Pokemon, LRF, OCZF and FBI-1) is a part of the POK (Kruppel and POZ/BTB) transcription factor family, and plays a major role during oncogenesis and cell differentiation (20). ZBTB7A was previously believed to be a proto-oncogene in various cancer types, including prostate cancer $(21,22)$. ZBTB7A is highly expressed in Hodgkin's lymphoma, as it represses the expression of AFR, a tumor suppressor (23). Nevertheless, a recent study conducted by Wang et al showed that it may have oncosuppressive functions in the case of prostate cancer upon PTEN loss (24). The prostate-specific transgene of Zbtb7a has no association with tumorigenesis, which is contrary to the previous hypothesis that it is an oncogene. Unexpectedly, the inactivation of $\mathrm{Zbtb} 7 \mathrm{a}$ accelerates Pten loss-induced prostate cancer progression. Mechanistically, the downregulation of retinoblastoma-associated protein by hyper-elevated Sox 9 was found to occur in Pten/Zbtb7a double-null prostate tumors (24); this resulted in Pten loss-induced cellular senescence being overcome (24). Therefore, Zbtb7a can also be identified as a PTEN context-dependent cancer gene that may have oncosuppressive and oncogenic functions in PTEN-null tumors.

\section{PTEN loss switches ARF function through sumoylation}

ARF is another transcript of the $A R F-I N K 4 a$ locus (CDKN2a) on human chromosome 9p21 (25). The CDKN2a locus encodes p14 ${ }^{\mathrm{ARF}}$ and p16INK4a, which are two cyclin-dependent kinase inhibitors (26). $C D K N 2 a$ deficiency may lead to susceptibility to carcinogen-induced tumors via antagonism of Mdm2-mediated p53 degradation (27). The canonical pathway of ARF induces senescence through degradation of MDM2, thereby stabilizing p53 (27).

Multiple studies have suggested that ARF has oncosuppressive and oncogenic functions. For instance, it has been shown that $19^{\text {Arf }}$ deficient mice develop various types of cancers, suggesting that ARF has oncosuppressive roles (26). On the other hand, a study by Chen et al (28) showed that p19 $9^{\text {Arf }}$ inactivation decreases tumorigenesis in PTEN-deficient prostate cancers, indicating the oncogenic role of ARF. Moreover, a number of previous studies have shown the oncogenic role of ARF in a genetic context-dependent manner $(29,30)$. However, the manner by which ARF function is switched from tumor suppressor to oncogene is unclear. In a previous study, we found that PTEN loss may be the switch during Zinc finger protein SNAI2 (SLUG)-mediated epithelial-mesenchymal transition (EMT). SLUG as a transcription factor can represses E-cadherin transcription to promote EMT in various cancers, including 


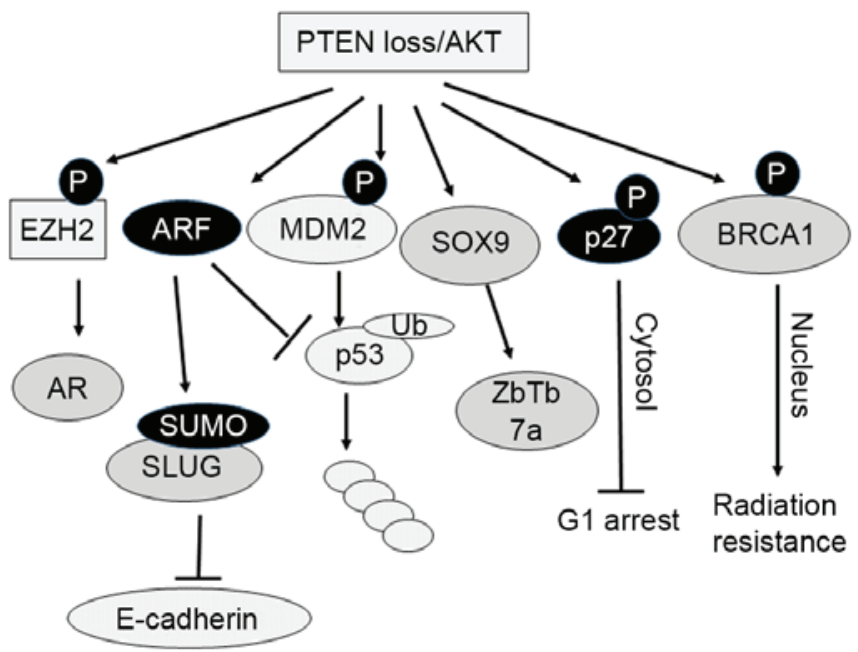

Figure 1. PTEN loss/AKT activation converts a subset of tumor suppressor proteins to oncogenic proteins or vice versa through multiple pathways. PTEN, phosphatase and tensin homolog; Akt, protein kinase B; EZH2, enhancer of zeste homolog 2; AR, androgen receptor; ARF, alternative reading frame 2; SUMO, small ubiquitin-like modifier; SLUG, ces-1-related zinc finger transcription factor; E-cadherin, epithelial cadherin; MDM2, mouse double minute 2; Ub, ubiquitin; SOX9, SRY-related HMG-box 9; ZbTb7a, zinc finger and BTB domain-containing 7A; BRCA1, breast cancer 1 , early onset; $\mathrm{P}$, phosphorylation.

prostate cancer (31). The mechanisms underlying these pathways are not described well, particularly when it comes to in vivo tumorigenesis during PTEN loss. In another previous study, we showed that p14 ${ }^{\mathrm{ARF}}$ (ARF in humans) stabilizes SLUG through sumoylation at lysine residue 192 (32). This stabilization results in the inhibition of E-cadherin in prostate cancer mouse models. On the other hand, p19 $9^{\text {Arf }}$ inactivation leads to the reduction of Slug levels, resulting in high E-cadherin expression (32). This inactivation delays the onset and progression of prostate cancer in Pten/Trp53 double null mice (32). This study suggested that PTEN loss may be the switch for ARF function from a p53-dependent tumor suppressor to an SLUG-EMT-dependent oncogenic protein. These novel findings may have implications for clinical research. Chemotherapeutic compounds that target ubiquitination to control cancer progression are currently in clinical trial. A similar approach can be used to develop inhibitors that will prevent cancer metastasis by blocking SLUG sumoylation. Moreover, it is possible to target ARF and make the treatment of prostate cancer more efficient.

\section{PTEN loss/AKT activation switches p27 from a tumor suppressor to an oncogene}

p27 is a tumor suppressor that represses cell cycle progression as an inhibitor of cyclin-dependent kinase (CDK) in the nucleus. However, the cytoplasmic localization of p27 has been found in a number of cancers and correlates with PTEN loss in prostate cancer (33). The cytoplasmic localization of p27 has been also found to be correlated with the activation of AKT in primary breast cancer samples (34). Mechanically, the AKT-mediated phosphorylation of p27 at threonine 157 determines cellular p27 translocation from the nucleus to the cytoplasm (34-36). As a consequence, cytoplasmic localized p27 inhibits G1 cell cycle arrest $(34,35)$. Thus, the PTEN loss/AKT activation pathway may switch p27 from a tumor suppressor to an oncogenic protein through phosphorylation mediated nuclear-cytoplasmic translocation.

7. PTEN loss/AKT activation switches BRCAl from a tumor suppressor to an oncogenic resistant gene in radiation therapy

BRCA1 is believed to act in the nucleus as a tumor suppressor that can repair DNA (37). The cytoplasmic localization of BRCA1 has been found in numerous cancers, where it results in the sensitization of DNA damage and increased apoptosis (38). Wild-type p53 promotes BRCA1 nuclear export upon irradiation (38). Consistently, dysregulated p53 induces the nuclear retention of BRCA1, which results in the resistance to radiation therapy in breast cancer cells (39). While p53 is regulated indirectly or directly by PTEN, PTEN loss may affect BRCA1 cellular localization and thereby switch the $B R C A 1$ from a tumor suppressor to an oncogenic resistant gene in radiation therapy. On the other hand, AKT has been found to phosphorylate BRCA1 to enhance its nuclear localization and stability, which results in the inhibition of radiation sensitivity (40). Thus, the PTEN loss/AKT activation pathway is most likely able to convert BRCA1 function.

In summary, with PTEN loss/AKT activation, functional switches of targets from oncogenes to suppressors and vice versa have been observed. These switches are associated with a subset of previously believed oncogenes or tumor suppressors (Fig. 1). It can be concluded that PTEN may be a powerful switcher, playing an oncosuppressive/oncogenic role in a context-dependent manner through multiple pathways.

\section{Conclusion and prospective outlook}

The results of this review suggest that PTEN inactivation may impact prostate cancer development significantly. This issue becomes particularly important in case of cancer treatment. For example, drugs targeting ZBTB7A may facilitate tumor growth if PTEN is inactivated. Therefore, a personalized medicine treatment approach should be taken into consideration, as gene deletions or mutations induce switches of oncogenic/tumor suppressive signaling, such as PTEN loss or mutations among individual patients.

Finally, with the development of methods for the transcriptional and genomic analysis of cancer cells, PTEN inactivation can potentially be used as a prognostic and diagnostic marker for various types of cancer, including, but not limited to, prostate cancer. As the understanding of the role of PTEN in the progression and initiation of cancer increases, this accumulating knowledge may be used for the design of novel therapeutics methods that will target PTEN-dependent pathways to treat different stages of prostate cancer.

\section{Acknowledgements}

This manuscript was supported in part by the Kazakhstan-China Collaboration Grant of Diagnosis and Precision Cancer Therapy. The authors would like to thank Nazarbayev 
University's grant support for the project, including the Social Policy Grant (to Dr Yingqiu Xie), SST Professional Development grant, and other under-review grants (IASANU Proposal grant and ORAU grant).

\section{References}

1. Gustafson S, Zbuk KM, Scacheri C and Eng C: Cowden syndrome: Semin Oncol 34: 428-434, 2007.

2. Blumenthal GM and Dennis PA: PTEN hamartoma tumor syndromes. Eur J Hum Genet 16: 1289-1300, 2008.

3. Feilotter HE, Nagai MA, Boag AH, Eng C and Mulligan LM: Analysis of PTEN and the 10q23 region in primary prostate carcinomas. Oncogene 16: 1743-1748, 1998.

4. Cairns P, Okami K, Halachmi S, Halachmi N, Esteller M, Herman JG, Jen J, Isaacs WB, Bova GS and Sidransky D: Frequent inactivation of PTEN/MMAC1 in primary prostate cancer. Cancer Res 57: 4997-5000, 1997.

5. Song MS, Salmena L and Pandolfi PP: The functions and regulation of the PTEN tumour suppressor. Nat Rev Mol Cell Biol 13 283-296, 2012

6. Ackler S, Ahmad S, Tobias C, Johnson MD and Glazer RI: Delayed mammary gland involution in MMTV-AKT1 transgenic mice. Oncogene 21: 198-206, 2002.

7. Bargonetti J and Manfredi JJ: Multiple roles of the tumor suppressor p53. Curr Opin Oncol 14: 86-91, 2002.

8. Di Cristofano A and Pandolfi PP: The multiple roles of PTEN in tumor suppression. Cell 100: 387-390, 2000.

9. Di Cristofano A, Pesce B, Cordon-Cardo C and Pandolfi PP: Pten is essential for embryonic development and tumor suppression. Nat Genet 19: 348-355, 1998

10. Zhou BP, Liao Y, Xia W, Zou Y, Spohn B and Hung MC: HER-2/neu induces p53 ubiquitination via Akt-mediated MDM2 phosphorylation. Nat Cell Biol 3: 973-982, 2001

11. Downward J: Mechanisms and consequences of activation of protein kinase B/Akt. Curr Opin Cell Biol 10: 262-267, 1998.

12. Mayo LD, Dixon JE, Durden DL, Tonks NK and Donner DB: PTEN protects p53 from Mdm2 and sensitizes cancer cells to chemotherapy. J Biol Chem 277: 5484-5489, 2002.

13. Chen Z, Trotman LC, Shaffer D, Lin HK, Dotan ZA, Niki M, Koutcher JA, Scher HI, Ludwig T, Gerald W, et al: Crucial role of p53-dependent cellular senescence in suppression of Pten-deficient tumorigenesis. Nature 436: 725-730, 2005.

14. Freeman DJ, Li AG, Wei G, Li HH, Kertesz N, Lesche R, Whale AD, Martinez-Diaz H, Rozengurt N, Cardiff RD, et al: PTEN tumor suppressor regulates p53 protein levels and activity through phosphatase-dependent and -independent mechanisms Cancer Cell 3: 117-130, 2003.

15. Lowe SW, Cepero E and Evan G: Intrinsic tumor suppression. Nature 432: 307-315, 2004.

16. Cao R, Wang L, Wang H, Xia L, Erdjument-Bromage H, Tempst P, Jones RS and Zhang Y: Role of histone H3 lysine 27 methylation in Polycomb-group silencing. Science 298: 1039-43, 2002

17. $\mathrm{Xu} \mathrm{K}, \mathrm{Wu} \mathrm{ZJ}$, Groner AC, He HH, Cai C, Lis RT, Wu X, Stack EC, Loda M, Liu T, et al: EZH2 oncogenic activity in castration-resistant prostate cancer cells is polycomb-independent. Science 338: 1465-1469, 2012.

18. Liu DC and Yang ZL: Overexpression of EZH2 and loss of expression of PTEN is associated with invasion, metastasis and poor progression of gallbladder adenocarcinoma. Pathol Res Pract 207: 472-478, 2011.

19. Zeng N, Yang KT, Bayan JA, He L, Aggarwal R, Stiles JW, Hou X, Medina V, Abad D, Palian BM, et al: PTEN controls $\beta$-cell regeneration in aged mice by regulating cell cycle inhibitor p16ink4a. Aging Cell 12: 1000-1011, 2013.

20. Vredeveld LC, Rowland BD, Douma S, Bernards R and Peeper DS: Functional identification of LRF as an oncogene that bypasses RASV12-induced senescence via upregulation of CYCLIN E. Carcinogenesis 31: 201-207, 2010.

21. Qu H, Qu D, Chen F, Zhang Z, Liu B and Liu H: ZBTB7 overexpression contributes to malignancy in breast cancer. Cancer Invest 28: 672-678, 2010.
22. Zhao ZH, Wang SF, Yu L, Wang J, Chang H, Yan WL, Zhang J and $\mathrm{Fu} \mathrm{K}$ : Overexpression of Pokemon in non-small cell lung cancer and foreshowing tumor biological behavior as well as clinical results. Lung Cancer 62: 113-119, 2008.

23. Aggarwal A, Hunter WJ III, Aggarwal H, Silva ED, Davey MS, Murphy RF and Agrawal DK: Expression of leukemia/lymphoma-related factor (LRF/POKEMON) in human breast carcinoma and other cancers. Exp Mol Pathol 89: $140-148,2010$.

24. Wang G, Lunardi A, Zhang J, Chen Z, Ala U, Webster KA, Tay Y, Gonzalez-Billalabeitia E, Egia A, Shaffer DR, et al: Zbtb7a suppresses prostate cancer through repression of a Sox9-dependent pathway for cellular senescence bypass and tumor invasion. Nat Genet 45: 739-746, 2013.

25. den Besten W, Kuo ML, Tago K, Williams RT and Sherr CJ: Ubiquitination of and sumoylation by, the Arf tumor suppressor. Isr Med Assoc J 8: 249-251, 2006.

26. Bardeesy N, Aguirre AJ, Chu GC, Cheng KH, Lopez LV, Hezel AF, Feng B, Brennan C, Weissleder R, Mahmood U, et al: Both p16(Ink4a) and the p19(Arf)-p53 pathway constrain progression of pancreatic adenocarcinoma in the mouse. Proc Natl Acad Sci USA 103: 5947-5952, 2006.

27. Ha L, Ichikawa T, Anver M, Dickins R, Lowe S, Sharpless NE, Krimpenfort P, Depinho RA, Bennett DC, Sviderskaya EV and Merlino G: ARF functions as a melanoma tumor suppressor by inducing p53-independent senescence. Proc Natl Acad Sci USA 104: 10968-10973, 2007.

28. Chen Z, Carracedo A, Lin HK, Koutcher JA, Behrendt N, Egia A, Alimonti A, Carver BS, Gerald W, Teruya-Feldstein J, et al: Differential p53-independent outcomes of p19(Arf) loss in oncogenesis. Sci Signal 2: ra44, 2009.

29. Humbey O, Pimkina J, Zilfou JT, Jarnik M, Dominguez-Brauer C, Burgess DJ, Eischen CM, Murphy ME: The ARF tumor suppressor can promote the progression of some tumors. Cancer Res 68: 9608-9613, 2008.

30. Khoo CM,Carrasco DR,Bosenberg MW,PaikJHand DepinhoRA: Ink4a/Arf tumor suppressor does not modulate the degenerative conditions or tumor spectrum of the telomerase-deficient mouse. Proc Natl Acad Sci USA 104: 3931-3936, 2007.

31. Hajra KM, Chen DY and Fearon ER: The SLUG zinc-finger protein represses E-cadherin in breast cancer. Cancer Res 62: 1613-1618, 2002.

32. Xie Y,Liu S,Lu W, Yang Q, Williams KD, Binhazim AA, Carver BS, Matusik RJ and Chen Z: Slug regulates E-cadherin repression via p19Arf in prostate tumorigenesis. Mol Oncol 8: 1355-3564, 2014.

33. Halvorsen OJ, Haukaas SA and Akslen LA: Combined loss of PTEN and p27 expression is associated with tumor cell proliferation by $\mathrm{Ki}-67$ and increased risk of recurrent disease in localized prostate cancer. Clin Cancer Res 9: 1474-1479, 2003.

34. Liang J, Zubovitz J, Petrocelli T, Kotchetkov R, Connor MK, Han K, Lee JH, Ciarallo S, Catzavelos C, Beniston R, et al: $\mathrm{PKB} / \mathrm{Akt}$ phosphorylates $\mathrm{p} 27$, impairs nuclear import of p27 and opposes p27-mediated G1 arrest. Nat Med 8: 1153-1160, 2002.

35. Shin I, Yakes FM, Rojo F, Shin NY, Bakin AV, Baselga J and Arteaga CL: PKB/Akt mediates cell-cycle progression by phosphorylation of p27(Kip1) at threonine 157 and modulation of its cellular localization. Nat Med 8: 1145-1152, 2002.

36. Viglietto G, Motti ML, Bruni P, Melillo RM, D'Alessio A, Califano D, Vinci F, Chiappetta G, Tsichlis P, Bellacosa A, et al: Cytoplasmic relocalization and inhibition of the cyclin-dependent kinase inhibitor $\mathrm{p} 27$ (Kip1) by PKB/Akt-mediated phosphorylation in breast cancer. Nat Med 8: 1136-1144, 2002.

37. Mullan PB, Quinn JE and Harkin DP: The role of BRCA1 in transcriptional regulation and cell cycle control. Oncogene 25: 5854-5863, 2006.

38. Wang H, Yang ES, Jiang J, Nowsheen S and Xia F: DNA damage-induced cytotoxicity is dissociated from BRCA1's DNA repair function but is dependent on its cytosolic accumulation. Cancer Res 70: 6258-6267, 2010.

39. Jiang J, Yang ES, Jiang G, Nowsheen S, Wang H, Wang T, Wang Y, Billheimer D, Chakravarthy AB, Brown M, et al: p53-dependent BRCA1 nuclear export controls cellular susceptibility to DNA damage. Cancer Res 71: 5546-5557, 2011.

40. Nelson AC, Lyons TR, Young CD, Hansen KC, Anderson SM and Holt JT: AKT regulates BRCA1 stability in response to hormone signaling. Mol Cell Endocrinol 319: 129-142, 2010. 